\title{
Alignment of the ATLAS Inner Detector
}

\author{
Mahsana Ahsan ${ }^{1}$ \\ University of Texas at Dallas \\ MS EC36, 800 West Campbell Road \\ Richardson, TX 75080, USA \\ E-mail: mahsancutdallas.edu
}

\section{Sebastian Fleischmann}

Physikalisches Institut, University of Bonn,

Nußallee 12, 53115 Bonn, Germany

E-mail: fleischmann@physik.uni-bonn.de

\section{On behalf of the ATLAS collaboration}

ATLAS is one of two general purpose detectors at the world's largest particle accelerator, the Large Hadron Collider (LHC), and has been designed to study proton-proton collisions at a center of mass energy $\sqrt{s}=14 \mathrm{TeV}$. While the LHC is currently operating at $\sqrt{s}=7 \mathrm{TeV}$, it collided proton beams at $\sqrt{s}=900 \mathrm{GeV}$ and $\sqrt{s}=2.36 \mathrm{TeV}$ between November 23 - December 16, 2009. ATLAS has an innermost charged particle precision tracking system for measurement of the trajectories of the charged particles emerging out of collisions. To achieve its physics goals the alignment of the tracking detector elements should be known to a precision of a few to few tenths microns. However, due to the large number of position sensitive devices, this constitutes a major challenge. This note presents the alignment approach and the results using the data collected from cosmic-rays and proton-proton collisions at $\sqrt{s}=900 \mathrm{GeV}$.

13th International Workshop on Advanced Computing and Analysis Techniques in Physics Research - ACAT 2010 Jaipur, India

February 22-27 2010 


\section{Introduction}

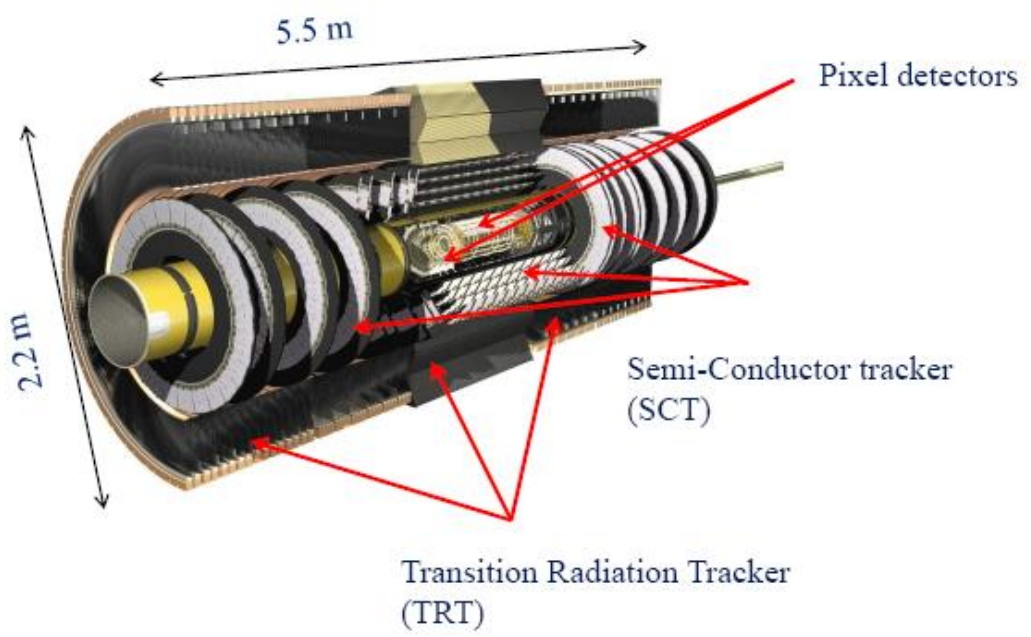

Figure 1: Schematic view of the ATLAS Inner Detector.

The Inner Detector (ID) of the ATLAS experiment at LHC [1], as shown in Figure 1 schematically, is comprised of three subsystems surrounding the interaction point: The Pixel detector (Pixels), the semi-conductor tracker (SCT) and the transition radiation tracker (TRT) [2]. The Pixel detector and the SCT use silicon-based detector technology, while the TRT is made of drift-tubes. The Pixel detector, which is the detector closest to the interaction point providing 2-dimensional position measurements, consists of three barrel layers and three endcap disks on either side of the barrel. The intrinsic spatial resolution of the Pixel silicon detector sensors with a nominal pixel size of $50 \mu \mathrm{m} \times 400 \mu \mathrm{m}$ is $14 \mu \mathrm{m} \times 115 \mu \mathrm{m}$ in the $r \phi \times z$ direction, where the $r \phi$ is the azimuthal direction and the $z$ coordinate is along the beam pipe. There are 1744 alignable Pixel modules. The SCT, consisting of four barrels and nine end-cap disks on either side of the barrel, has 4088 modules composed of two sets of daisy-chained silicon strip sensors of $80 \mu \mathrm{m}$ pitch laid back-to-back with a $40 \mathrm{mrad}$ stereo angle. It provides a spatial resolution of $17 \mu \mathrm{m}$ in the $r \phi$ direction. The outermost ID tracking system, the TRT, is a gaseous system of straw tubes with anode wires, operated with a mixture of $\mathrm{Xe}, \mathrm{CO}_{2}$, and $\mathrm{O}_{2}$. The straws are arranged in three cylindrical layers of 96 barrel modules and 14 disks on either side of the TRT barrels. There are 176 alignable TRT modules with an intrinsic spatial resolution of $130 \mu \mathrm{m}$ in the $r \phi$ direction. The entire ID system lies inside a superconducting solenoid providing a $2 \mathrm{~T}$ magnetic field.

The ID tracking system is used to reconstruct tracks and their vertices by measuring charge deposition and local hit positions. The alignment of the modules should be known with high accuracy to exploit the intrinsic resolution of the precision tracking devices. This is critical for important physics measurements such as the $W$ mass measurement and heavy-flavor tagging for discovery searches. The position of the modules in the most sensitive direction should be known with a precision of about $7 \mu \mathrm{m}$ for the Pixels, $12 \mu \mathrm{m}$ for the SCT, and $30 \mu \mathrm{m}$ for the TRT, in order not to degrade the track parameter resolutions by more than $20 \%$. For the most precise physics, it is desirable to align to a few to few tenths of microns level. Each detector element has three translational and three rotational degrees of freedoms (DoFs). It is very challenging to align a large tracking system with 34992 DoFs for the silicon detectors, and 960 DoFs for the TRT. This requires the development of sophisticated techniques to handle the large number of DoFs. 


\section{ID Alignment at ATLAS}

Mechanical and optical surveys of the silicon detectors were performed before installing the components into the detector, as described in [3] and [4]. A precise geometrical survey was possible only for the Pixel detector sectors at the time of installation. The Pixel detector survey data can be used with the track-based alignment algorithms, discussed in Section 2.1, to set further constraints on the alignment parameters. The assembly tolerances are $O(100) \mu \mathrm{m}-$ $\mathrm{O}(1000) \mu \mathrm{m}$ for the larger structures such as the silicon barrel layers and end-cap disks, and $\mathrm{O}(100) \mu \mathrm{m}$ for the silicon modules.

The SCT is equipped with frequency scanning interferometry (FSI) [5], which is an optical alignment monitoring system consisting of 842 grid-line interferometers, a reference interferometer, and a tunable laser for frequency scanning to perform quasi-real time position measurements. This system is currently in the commissioning phase and has not yet actually been used for the detector alignment.

\subsection{Track-based alignment}

The track-based alignment is based on minimizing the $\chi^{2}$ of the track residuals. The track residual vector $\boldsymbol{r}(\boldsymbol{a}, \boldsymbol{\pi})$, depending on alignment parameters $\boldsymbol{a}$ and track parameters $\pi$, is defined as the distance between the measured hit position and the extrapolated track intersection in the module plane. The $\chi^{2}$ is constructed from the residuals as

$$
\chi^{2}=\sum_{\text {tracks }} \boldsymbol{r}^{T} V^{-1} \boldsymbol{r},
$$

where $V$ is the covariance matrix, and the sum is over the selected tracks in many events. Minimizing the $\chi^{2}$ with respect to the alignment parameters gives:

$$
\delta \boldsymbol{a}=-M^{-1} \times \mathrm{U},
$$

where $U=\sum_{\text {tracks }} \frac{d \boldsymbol{r}^{T}}{d \boldsymbol{a}} V^{-1} \boldsymbol{r}$ and $M=\sum_{\text {tracks }} \frac{d \boldsymbol{r}^{T}}{d \boldsymbol{a}} V^{-1} \frac{d \boldsymbol{r}}{d \boldsymbol{a}}$,

and $\frac{d r}{d \boldsymbol{a}}=\frac{\partial r}{\partial \boldsymbol{a}}+B$, where $B=\frac{\partial r}{\partial \pi} \frac{d \pi}{d \boldsymbol{a}}$.

There are four track-based alignment algorithms developed at ATLAS: Global $\chi^{2}$ algorithm (GX2), Local $\chi^{2}$ algorithm (LX2), Robust algorithm (RA), Pixel standalone algorithm (PSA). While all the algorithms produce consistent results, the GX2 is the baseline algorithm. The GX2 algorithm introduces correlations between modules via the common track through the implicit track refit represented by the $B$ term, while the LX2 and RA ignore this term. The LX2 and RA differ themselves in the definition of track residual and in the treatment of the errors used in the covariance matrix. The RA algorithm has been recently modified from its original context to extract alignment corrections for translation in the module local $Z$ direction and rotation around the local $Z$ axis, in addition to the alignment corrections for translations in the local $X$ and $Y$ direction. The local coordiantes of modules are defined such that the $X$ and $Y$ orthogonal coordinates lie in the module plane, where $Y$ is along (perpendicular to) the beam direction for the barrel (end-cap disks), and the $Z$ coordinate is normal to the module plane. The improved RA algorithm produces results comparable to that of the GX2 and LX2 algorithms. The PSA algorithm has been developed for the Pixel detector alignment only and provides a consistency check with other algorithms. The TRT alignment is performed using an approach similar to the GX2. A more detailed description of these algorithms can be found at [6-9].

The misalignments of the detector are determined at three major levels, namely Level 1, Level 2, and Level 3. Level 1 corresponds to larger structures, such as the Pixel detector as a 
whole, the SCT barrel, and the SCT end-caps, reducing the scale of the problem to 24 DoFs. At Level 2, three Pixel barrel layers, three Pixel end-caps on either side of barrel, and four SCT barrel layers and nine end-caps on either side are considered as alignable structures, leading to alignment in a few hundred DoFs. At Level 3, individual silicon module misalignment is determined. In order to remove larger misalignments, silicon modules are aligned initially at Level 1, followed by Level 2 and Level 3, and the steps are repeated to minimize the effects from systematic shifts or rotations of smaller structures. The TRT alignment is done only at Level 2. The extraction of the alignment constants at Level 1 and Level 2 involves solving full matrix inversion, while at Level 3 only sparse matrix inversion is considered due to the huge scale of the problem. Alignment algorithms are operated iteratively on the reconstructed and refitted tracks for all these levels. It takes $\mathrm{O}(100)$ processors, depending on the size of the input data set, and full convergence requires $\mathrm{O}(10)$ iterations.

The ID alignment operation procedure at ATLAS is as follows. First, a set of alignment corrections is obtained for the silicon using the track-based algorithms and the 'center-ofgravity (CoG)' corrections is applied to remove global detector movements with respect to the interaction point. This correction transforms the $\mathrm{CoG}$ of the newly aligned silicon detector back to the origin of the ATLAS coordinate system. Then a set of alignment constants are produced for the TRT with respect to the aligned silicon geometry. The CoG correction is applied finally for the whole ID system. The final set of alignment constants at this stage are validated by looking at the track-hit residuals, track parameter distributions and other physics objects.

\subsection{Weak Modes}

There could be certain global deformations present in the detector that leave the track-hit residuals and track $\chi^{2} / \mathrm{DoF}$ unchanged. Such deformations are known as 'weak modes', and these produce bias in the fitted track parameters. The most dangerous ones which have a high impact on physics are known as 'Curl', 'Telescope', 'Elliptical', and 'Twist' modes. The Curl mode, biasing the track curvature, is a global phi rotation of modules which increases as a function of radius. The Telescope mode is the global $z$ translation of the modules which increases radially and biases the track pseudorapidity $\eta$. The Elliptical mode is a radial distortion of the modules varying with global $\phi$ and produces a bias in the mass of reconstructed objects. The Twist mode is a global $\phi$ rotation of modules which increases with global $z$, causing an $\eta$-dependent track curvature bias. The MC studies performed at ATLAS for these systematic deformations are illustrated and discussed in detail elsewhere [10].

Track-based alignment methods need additional constraints, e.g. a common vertex constraint, an energy-to-momentum ratio constraint, and alignment parameter constraints, to remove the weak modes. The cosmic-ray and beam halo events can serve to tackle weak modes in the barrel regions and the end-cap regions, respectively.

\section{Alignment with cosmic-ray and proton-proton collision data}

ATLAS has been collecting cosmic-ray data since September, 2008. LHC proton-proton collisions at $\sqrt{s}=0.9 \mathrm{TeV}$ and $2.36 \mathrm{TeV}$ have been recorded by ATLAS with the full tracking system operational in December 2009. Early ID alignment has been performed using the Autumn 2008 cosmic-ray data comprising about 5 (2.6) million tracks without (with) solenoid magnetic field. Of these, about 0.2 million tracks have hits in the Pixel and SCT detectors in each magnet configuration. Figure 2 illustrates the residual distribution in $r \phi$ for the silicon and the TRT as found in cosmic-ray data after an early aligned geometry was produced using cosmic-ray tracks [11]. In these distributions, the tracks are required to have transverse momentum $p_{T}>2 \mathrm{GeV}$, impact parameter in the transverse plane $\left|d_{0}\right|<50 \mathrm{~mm}$, and impact parameter in the longitudinal plane $\left|z_{0}\right|<400 \mathrm{~mm}$. The open square and filled circle distributions represent the cosmic-ray data for nominal geometry and track-based aligned 
geometry, respectively, while the open circle distributions are representative of the MC simulation for perfect geometry. The residuals obtained in data are approaching the MC simulations, showing that the misalignments are well understood.

Tracks from proton-proton collisions at $\sqrt{s}=0.9 \mathrm{TeV}$ were combined with the cosmic-ray tracks to re-align the ID. The collision data sample is dominated by low-momentum tracks from soft interactions. There were about 0.5 million tracks that have been used for the alignment. The tracks from proton-proton collisions greatly improve the alignment in the endcap regions. The end-cap regions could not be aligned well using only the earlier cosmic-ray data due to the lack of tracks at normal incidence to the disk modules. Figure 3 illustrates the residual distributions for the Pixel and SCT barrels and end-caps in collision data for preliminary aligned geometry compared with the distribution from MC simulation for perfect alignment. The tracks are required to have $p_{T}>2 \mathrm{GeV},\left|d_{0}\right|<10 \mathrm{~mm}$, and at least six silicon hits.
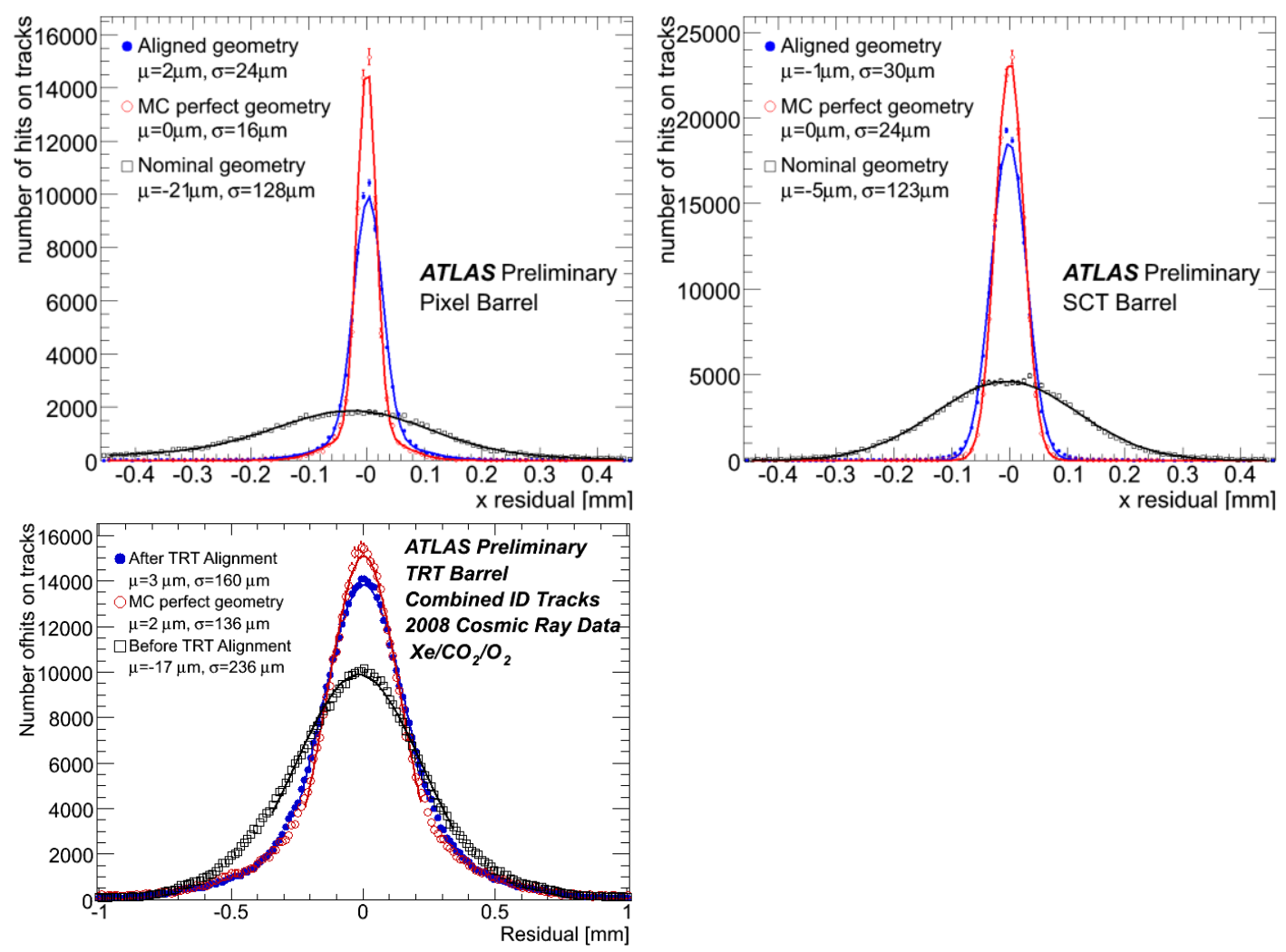

Figure 2: Distributions of track-hit residuals in $r \phi$ as obtained in cosmic-ray data for the Pixel barrel, the SCT barrel, and the TRT barrel are shown in the top left, the top right, and the bottom plots, respectively [10]. A double gaussian fit is performed in the range $\pm 0.5 \mathrm{~mm}$ to the distributions shown in the top left plot, and the mean $\mu$ and sigma $\sigma$ parameters of the narrower gaussian are reported. For the top right plot the distributions are fit with single gaussian in the range $\pm 0.5 \mathrm{~mm}$, and $\mu$ and $\sigma$ parameters of the gaussian are reported. A single gaussian fit is performed to the distributions shown in bottom plots, the fit is iterated until the range corresponds to \pm 1.5 times $\sigma$, and their mean $\mu$ and sigma $\sigma$ are reported. The alignment geometry obtained from the GX2 algorithm are used here.

\section{Conclusions}

Various alignment techniques employed at ATLAS have been performing very well. The track-based alignment has been performed using tracks from both cosmic-ray data and proton- 
proton collisions at $\sqrt{s}=0.9 \mathrm{TeV}$. The widths of the residual distributions are approaching those of simulations with a perfect knowledge of the geometry. As the LHC is currently operating at $\sqrt{s}=7 \mathrm{TeV}$, more collision data will be collected up to 2011. This will greatly help to further improve the ID alignment to achieve the ATLAS physics goals.
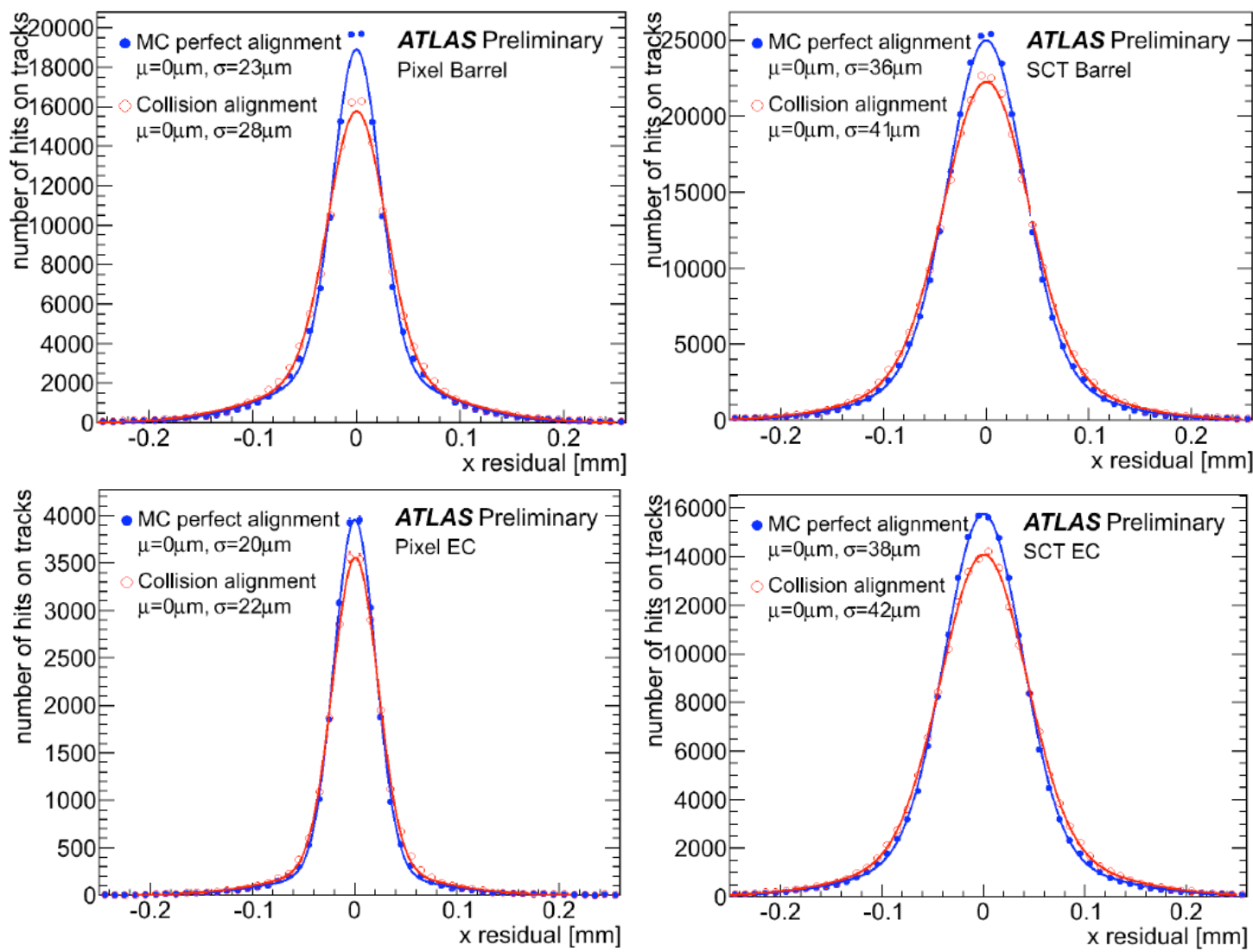

Figure 3: Distribution of track-hit residuals in $r \phi$ as obtained in proton-proton collision data after realigning by combining the collision and cosmic-ray data. The distributions for the Pixel and SCT barrels are shown in the top left and top right, and for the Pixel and SCT end-caps are shown in the bottom left and bottom right, respectively. A double gaussian fit to track-hit distributions is performed in the range $\pm 0.3 \mathrm{~mm}$, and the mean $\mu$ and the sigma $\sigma$ of the narrower gaussian are reported in the figures. The alignment geometry obtained from the GX2 algorithm is used here.

\section{References}

[1] L. Evans, P. Byrant, LHC Machine, JINST 3(2008) S08001.

[2] G. Aad et al. [The ATLAS Collaboration], The ATLAS Experiment at the CERN Large Hadron Collider, JINST 3 (2008) S08003.

[3] T.Golling, ATL-INDET-PUB-2006-001 (2006).

[4] A. Andreazza et al., ATL-INDET-PUB-2008-012 (2008).

[5] P.A. Coe, D.F. Howell, R.B. Nickerson, Meas. Sci. Technol. 15 (2004) 2175-2187; S. Gibson et al., Opt. Las. Eng. 43, Issue 7 (2005) 815-831.

[6] P. Bruckman de Renstrom, A. Hicheur and S. Haywood, ATL-INDET-PUB-2005-002 (2005).

[7] R. Härtel, Diploma thesis, TU München, Germany, (2005).

[8] T. Göttfert, Diploma thesis, Universität Würzburg, MPP-2006-118 (2006).

[9] F. Heinemann, ATL-INDET-PUB-2007-011 (2007).

[10] G. Aad et al. [The ATLAS Collaboration], ATLAS-PHYS-PUB-2009-080 (2009). 
[11] D. Kollar, J. Phys.: Conf. Ser. 219 (2010) 032046. 